\title{
Grasped Element Position Recognition and Robot Pose Adjustment during Assembly
}

\author{
K. Iturralde $1^{\mathrm{a}}$, T. Kinoshita $2^{\mathrm{b}}$, T. Bock $3^{\mathrm{a}}$ \\ ${ }^{a}$ Chair of Building Realization and Robotics, Technical University of Munich, Germany \\ ${ }^{b}$ Division of Human Mechanical Systems and Design, Graduate School of Engineering, Hokkaido University, Japan \\ E-mail: kepa.iturralde@br2.ar.tum.de
}

\begin{abstract}
-
Upgrading building envelopes with fully prefabricated 2D modules requires high accuracy during the manufacturing process with tolerances lower than $1 \mathrm{~mm}$. In the research described in this paper, computer designed and accurately manufactured objects have been assembled with robotic arms. However, in previous phases, it was detected that during the assembly process, the placement differed from the planned location due to undesired deviations of the object while being grasped and placed by the robotic tool. The experiments presented in this paper imply correcting this deviation by localizing the grasped object's position and recalculating the path and final pose of the assembly process. For localizing the deviated grasped object's location, an intermediate pose was planned. During this pose, the location of the grasped object was estimated by two different means. For the first solution, visual markers have been placed on a known corner of the objects and these have been recognized with a camera. For the second solution, the coordinates of the objects were measured by a digital theodolite. The location of the deviated object was calculated and compared to the planned location so the robot could divert from its original path. The measurable parameter in the experiments was the assembly accuracy. The results in the two experiments have been analyzed and compared. According to the results, the solutions could be implemented not only in the factory, but at on-site processes as well.
\end{abstract}

Keywords deviation, localization, estimation, adjustment

\section{Introduction}

The existing building stock's envelope capabilities need to be upgraded in order to achieve zero-energy consumption [1]. In an ongoing research project named BERTIM [2], timber based prefabricated 2D modules with embedded renewable energy sources (RES) are fixed onto existing building façades in order to improve the insulation of the building and generate energy. According to the objectives of the research project, these 2D modules should be fully prefabricated, which means that no rework should be necessary after fixing the modules onto the façade. As a consequence, on-site installation time is reduced. These fully prefabricated modules need to achieve waterproof and airtight conditions while ensuring fitting ducts [2]. This requires an accurate manufacturing of the modules with very low tolerances of up to $1 \mathrm{~mm}$. In 2D module manufacturing with timber-frames, traditional off-site techniques [3] don't fulfil the demanded accuracy, where tolerances can reach up to $10 \mathrm{~mm}$. In brief, current marketed processes are based on precut studs and beams, which are placed in a carpentry bench according to their position. Afterwards, studs and beams are nailed and the frame is compiled. In the next step, OSB boards are placed and nailed onto the timber-frame and, finally, the OSB boards are cut by a CNC bridge crane.

There are several reasons for such inaccuracies that were pointed out in previous phases of the research [3]. As a solution to these inaccuracies, it was proposed that all the objects of the prefabricated modules should be contoured in a CNC machine and then robotically assembled to create a façade module as shown in Figure 1. In this sense, there is experience in the manufacturing process of $\mathrm{CNC}$ routed timber objects [4]. Moreover, there are some experiences that link the design and the robot thanks to parametric design tools [5]. However, besides some exceptions [6], the assembly of studs and boards is mainly achieved manually. The robotic assembly of prefabricated modules is still a process which faces some challenges related to accuracy as well. The remainder of this paper is to define and test a solution related to overcome accuracy issues with the robotic assembly processes. 


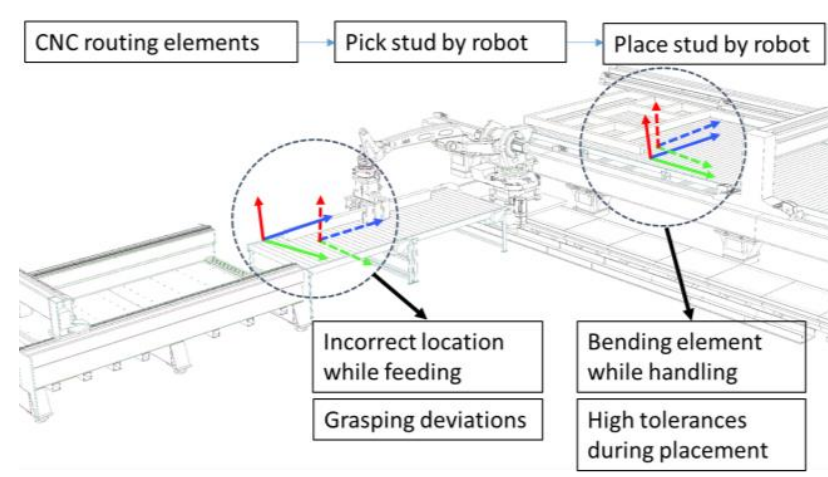

Figure 1. Scheme for a fully robotized assembly of prefabricated timber frames

In Figure 1 it is shown an scheme based on $\mathrm{CNC}$ routed elements that are robotically placed into an automated multi-function bridge for the assembly. In previous phases of the research, it was detected that deviations occur mainly while grasping and placing the timber elements by the robot.

\section{Challenges of robotic assembly of fully prefabricated (timber-framed) modules}

30 years ago, Kodama et al. [7] reported deviations during the grasping and placing of construction blocks while building a wall. These blocks were specially designed for facilitating the assembly process following the robot oriented design (ROD) concept [8]. However, the deviation of the wall built was considerable. Gambao et al.[9] also faced similar issues during the erection of a wall with robots during the ROCCO project. In more recent research projects, ordinary bricks were used to build parametrically designed walls. Bonwetsch [10] reported deviations of up to $10 \mathrm{~mm}$ compared to the desired location. Similar deviations happened in a research project that assembled timber profiles for building structures and, for this case, Willmann et all [6] suggested sensor feedback mechanisms in order to allocate the grasped object as well as the assembled module. In the researches explained by Willmann and Bonwetsch [6, 10], the objects to be assembled don't present any special joining system such as ROD, which in turns does not facilitate the allocation of the parts or elements with each other. On the other hand, some timber-framing machine builders offer the possibility of robotizing the assembly process of boards (not timber frame elements such as studs and mullions). However, as can be seen in reference [11], the grasped object tends to bend, which jeopardizes the exact placement of the object.

Therefore, it can be stated that the current robotic tools aren't as precise as desired in the assembly process of prefabricated modules. The accuracy of the robot's grasping is not guaranteed. This lack of precision increases especially when working in unstructured environments where the grasped objects aren't placed in a known location. Besides, the variety of design of the prefabricated modules hinders an automated programming of the robot's grasp and path planning.

During the research project explained in this paper, some tests were already achieved before [12]. A simulation was defined and some problems were intercepted (see also Figure 1):

- Incorrect placement of objects during feeding

- Deviations while grasping the objects

- In the case of some objects, especially boards, the object tends to bend and, therefore, the final position might not be adjusted

- Tolerances due to robotic arms calibration and accuracy during placing

As stated previously, it is necessary to recognize the location of the grasped or handled object to be assembled. In this sense, robotic assembly in construction can take advantage of concepts such as measurement assisted assembly (MAA). Maropoulos et al. [13] determined a solutions that enable a more predictive and flexible assembly process by using active tooling and closed loop control. This concept was mainly developed for complex and large-scale assembly processes such as in the aviation industry, but it can be used for the construction industry as well. Following these ideas, Druot et al. [14] applied the MAA for high accuracy aerospace assembly with robots with optimal results.

During the assembly process with robots, an adjustment of the robot's path is necessary. There is already literature where robots' paths can be adjusted depending on the feedback that the robot receives from different data acquisition and sensing devices and there are also some experiences in robotic assembly that can be found in the literature. Nottensteiner et al. defined a system to recognize objects and plan the assembly process by using two robotic arms [15]. In the research carried out by Feng et al. [16], markers were used for localizing objects and defining a plan for the assembly of parametrically designed walls. Finally, Lundeen et al. [17] used optical marker on top of the end effector to estimate the pose of articulated excavators.

\section{$2.1 \quad$ Objective}

The main research objective presented in this paper is to adjust the robotic assembly path depending on the location of the grasped element. Subsequently, the research question is if element location recognition and object path adjustment improve the deviations and 
displacements generated while grasping the object during the assembly processes. The objective is to increase accuracy on the final pose for achieving the assembly of a timber-frame with $\mathrm{CNC}$ routed elements. For achieving that goal, it has been necessary to use visual systems that recognize the position of the grasped element and accordingly correct the deviation by adjusting the path of the robot.

\section{Tests in laboratory environment}

The works carried out during the experimentation phase consisted on assembling a mockup that resembled a timber-frame. The objects of the mock-up were made or fabricated by a 3D printer (German RepRap X400@) using PLA filament (Polylactide PLA from German RepRap (C) as an additive material. The objects were dovetailed as can be seen in Figure 2 in order to facilitate the placement by the robot.

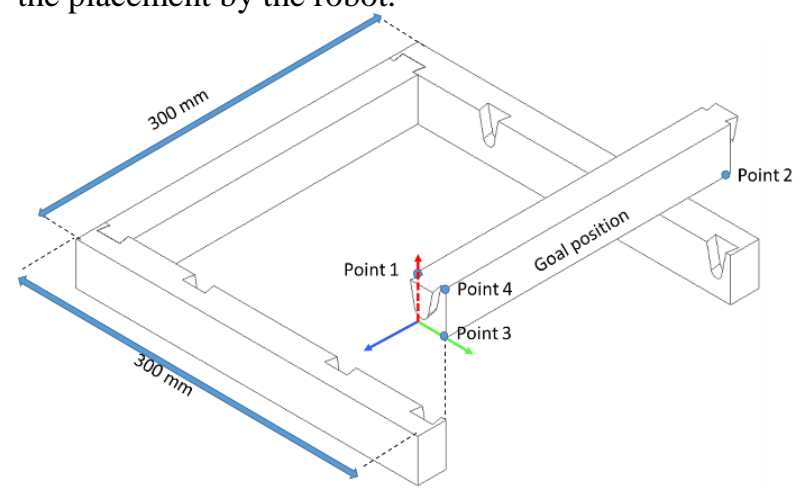

Figure 2. Prefabricated module mockup used for the assembly in laboratory environment experiments.

The robot was placed in a referenced/known location with respect to the assembled module. The objects were also in known locations. The size of the mock-up was $300 \mathrm{~mm} * 300 \mathrm{~mm} * 35 \mathrm{~mm}$, which is about ten times smaller than a conventional prefabricated timber-frame module.

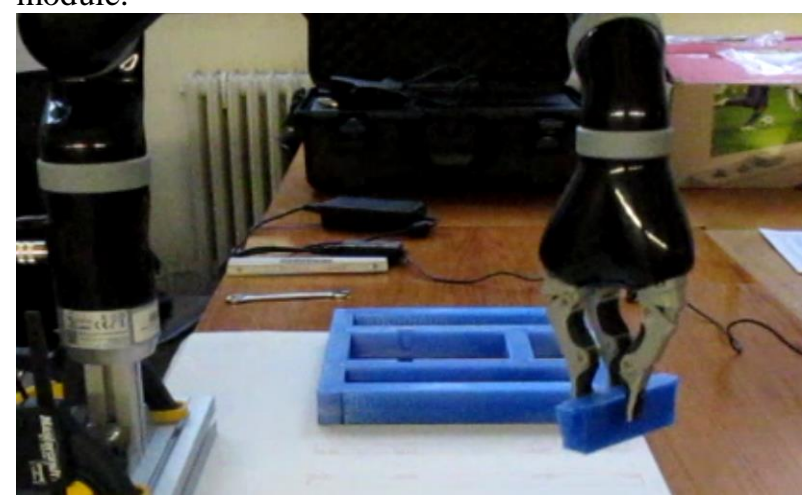

Figure 3. Assembly process carried out by the
Kinova Jaco $\mathbb{R}$ robotic arm.

The robot used for the assembly process was a Kinova JacoR (six Degrees of Freedom) robotic arm (see Figure 3 ). It is noteworthy that the scale of the mock-up and the functionalities of the robot don't appear to reflect the reality of the assembly of the prefabricated module or that of the building industry. However, the materials and the robot used for the test reflect a worst-case scenario regarding deviations. On the one hand, the accuracy of the end-effector (hand type) of the Kinova Jaco $\mathbb{R}$ is not appropriate for grasping cubicle objects and, therefore, the deviations are considerable and appear exaggerated when compared to a gripper that is more adequate for such conditions. These "large" deviations are "good"; it is assumed that the robot and the mockup are suitable for this test and carrying out the adjustment of such grasping inaccuracies while picking and placing objects. In Table 1 , the materials and devices used during the experimentation are defined.

Table 1 : Equipment, materials and resources used

\begin{tabular}{lc} 
Computer processor & Intel CORE i7 8th Gen \\
Robotic arm & Kinova Jacoß \\
Controller & ROS \\
Path planning & MoveIt! \\
Camera & Logitech C170@ \\
Digital theodolite & Leica 3D Disto@ \\
Light source & LED lamp \\
\hline
\end{tabular}

The protocol for the experimentation follows a linear process. This linear process is included in a broader integrated workflow that spans from the data acquisition of the existing building to the assembly process of the prefabricated modules (it must be recalled that the prefabricated modules are conceived for building renovation [3]). The protocol for this experimentation starts by gathering the information that parametric software named Dynamo@ [18] generates, which is a list with the coordinates of the location of each of the objects. In ROS-MoveIt! environment, the robot reads the list and plans the motion and starts executing the plan. During the execution of the planned operations, there is a pause in the path. This paused pose's objective is to facilitate the recognition of the location of the grasped element. Three tests were carried out during the experimentation phase. The first test was achieved without applying any deviation correction. The second test was achieved by the use of ArUco markers for localizing the grasped object's place. The third test was achieved by using a digital theodolite or total station for localizing the key point coordinates of the object. Each test was repeated five times. Once the grasped object's location was determined, the robot modified its position in order to get closer to the planned location of the object. All three tests finalized 
with a goal position where the location of the grasped object was measured in order to define the accuracy obtained in each test. Four points were measured as it is shown in Figure 2 and Table 2: point 1, point 2, point 3 and point 4 .

Table 2 Planned location for point 1, 2, 3, and 4(mm).

\begin{tabular}{llll}
\hline Name & Position X & Position Y & Position $\mathrm{Z}$ \\
\hline Point 1 & 0.0 & 25.0 & 0.0 \\
Point 2 & 0.0 & 25.0 & 37.5 \\
Point 3 & 0.0 & 0.0 & 37.5 \\
Point 4 & -250.0 & 25.0 & 0.0 \\
\hline
\end{tabular}

In all tests, the robot was positioned with respect to a reference coordinate, in other words, the position of the robot was independent of the location of the assembly module.

The deviation of positions is obtained by the equations as follows:

$$
\begin{gathered}
r_{i}=\sqrt{\left(x_{n i}-x_{0}\right)^{2}+\left(y_{i}-y_{0}\right)^{2}+\left(z_{i}-z_{0}\right)^{2}} \\
\bar{r}=\frac{\sum_{i=1}^{N} r_{i}}{N} \\
\sigma_{k}=\sqrt{\frac{\sum_{i=1}^{N}\left(r_{i}-\bar{r}\right)}{N}}
\end{gathered}
$$

where $N$ is number of trials, $\left(x_{0}, y_{0}, z_{0}\right)$ is the planned location and $\left(x_{i}, y_{i}, z_{i}\right)$ is the accomplished location during the $i$ number of trials. The accuracy of the test is shown by the equation as follows:

$$
\delta_{k}=\sqrt{\frac{\sum_{i=1}^{N} r_{i}}{N}}
$$

\subsection{Test without any deviation correction}

This experimentation was carried out in order to determine the benchmark or the "normal" capabilities of the robot. The protocol is a process without any iterative step as shown in Figure 4, and no correction or adjustment was applied.

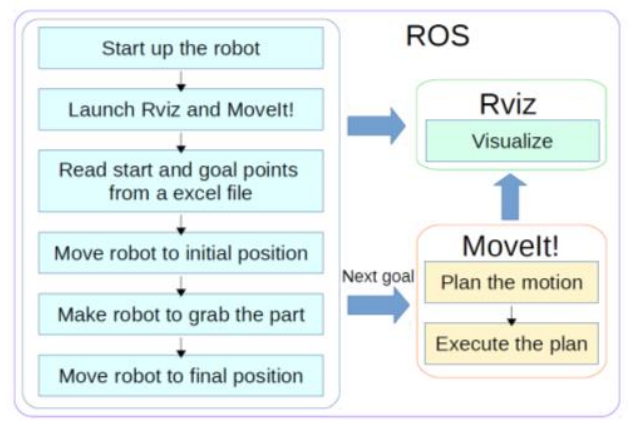

Figure 4. Protocol of the assembly process for the test without any deviation correction.
The path planning and the grasping was determined in advance by the data generated for the parametric software. In Figure 5, the five different localitions of the object on the final pose of the robot are shown in red. In green, the planned location of the object are shown. In Table 3, the average point coordinates of the five different locations can be seen.

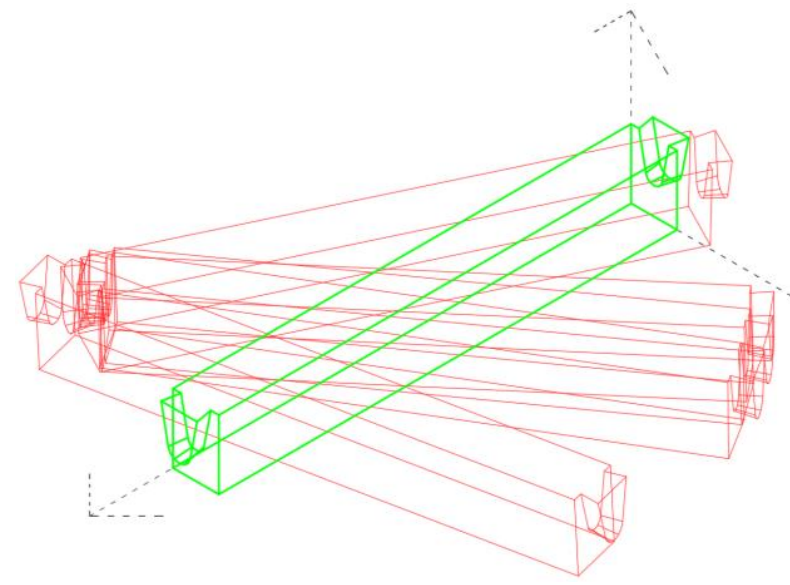

Figure 5. Goal position results without using any deviation adjustment.

The results of the pose without any pose deviation, as expected, show a high deviation in comparison to the planned location of the object, where distances reach up to $136,86 \mathrm{~mm}$ in Point 2.

Table 3 Absolute location in the test without any deviation correction and distance from planned location (mm).

\begin{tabular}{lrrrr}
\hline Name & Position X & \multicolumn{1}{c}{ Position Y } & Position Z & Distance \\
\hline Point 1 & $-65,20$ & $-106,50$ & 14,90 & 147,53 \\
Point 2 & $-47,70$ & $-94,10$ & $-10,10$ & 136,84 \\
Point 3 & $-47,10$ & $-93,00$ & $-1,80$ & 111,41 \\
Point 4 & $-188,60$ & 99,20 & $-22,60$ & 98,93 \\
\hline
\end{tabular}

As can be observed in Table 3, the results are considerably poor and impede the assembly of the mockup with deviations higher than $100 \mathrm{~mm}$. Therefore, these results show a worst-case scenario regarding deviations that need to be improved upon in the next two tests.

\subsection{Test with Open CV and ArUco markers.}

This test was based on the capabilities of Open CV [19] for recognizing the so-called ArUco markers. It states on their official website [19] "OpenCV (Open Source Computer Vision Library) is an open source computer vision and machine learning software library". In this test, two types of markers were used according to their functionality. First, a set of markers was placed onto the grasped element so that the pose of the center of it could be obtained if any of the markers were detected. The other marker was fixed on the working table as a 
reference for the coordinates of the robot and the assembled module. The marker on the working table was used as a reference for the positioning.

During this test, an iterative step was defined in order to check and correct the deviation as explained in Figure 6. This iterative step improved the adjustment of the goal position. The camera was calibrated by using OpenCV and the chess board square placed in front of the Jaco robot. The corner of this square was used as a Reference coordinate, as it is shown in Figure 7.

The detection of the markers was not without issues e.g. it was affected by insufficient lighting. Furthermore, the occlusion of the markers caused the grasped object to not be recognized. When the markers were inclined too much away from the camera, it was difficult to detect them. In addition, $\mathrm{z}$-axis flipping sometimes occurred [20]. This problem was prevented by using several markers and by accepting the majority decision.

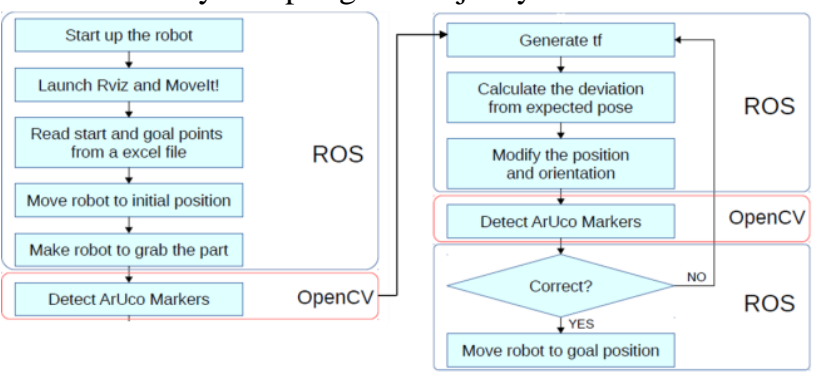

Figure 6. Protocol of the assembly process for the test with the ArUco markers.

However, due to specific conditions such as grasped point or brightness, only one marker was detected and $\mathrm{z}$ axis flipping was found to occur occurred.

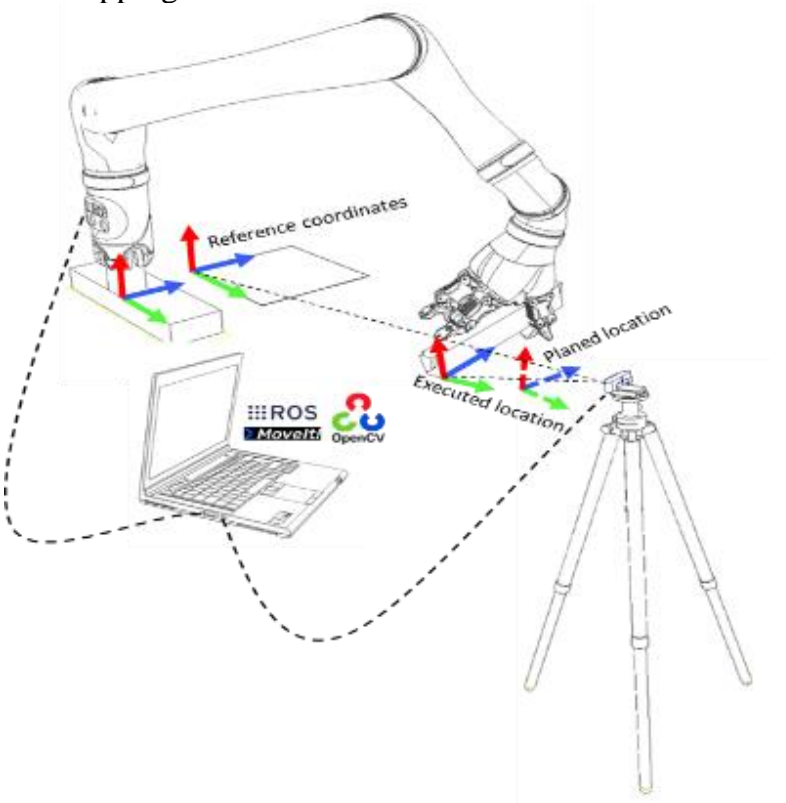

Figure 7. Scheme of the recognition of the grasped object by using the ArUco markers.

In Figure 7, the scheme on the left shows the relative simplicity of the system. The camera needed to be placed on the point where the markers on the object and the reference marker can be seen at the same moment. The results; however, improve the final position location considerably.

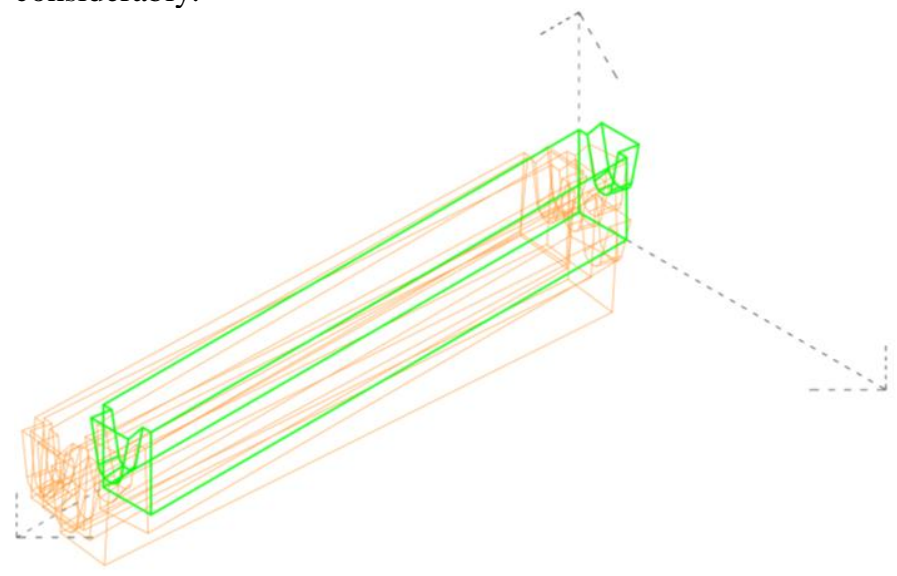

Figure 8. Goal position results using markers.

In Figure 8, the five different locations of the object on the final pose of the robot are presented in orange. In green, the planned location of the object is shown.

Table 4: Absolute location in the test with the markers and distance from planned location $(\mathrm{mm})$.

\begin{tabular}{lrrrr}
\hline Name & Position X & Position Y & Position Z & Distance \\
\hline Point 1 & 11,50 & 11,80 & 9,40 & 19,87 \\
Point 2 & 11,40 & 10,10 & 1,90 & 40,24 \\
Point 3 & 13,20 & $-10,30$ & 28,20 & 19,15 \\
Point 4 & $-238,10$ & 18,10 & $-14,20$ & 19,77 \\
\hline
\end{tabular}

In Table 4, the results show deviations smaller than those that were presented in the test in sub-chapter 3.1. Similar to the test in sub-chapter 3.1, in Table 4, the average point coordinates of the five different locations are shown.

\subsection{Test with the digital theodolite.}

In this test, during the intermediate pose, the objects were recognized by localizing three points of the object. This test further requires that a human operator recognizes the location of the grasped object points by the Leica 3D Disto@.

During this test, an iterative step was defined as well as in the previous test, in order to check and correct the deviation as explained in Figure 9. In all five tests, the same three points of the element's corner were measured in the same order. 


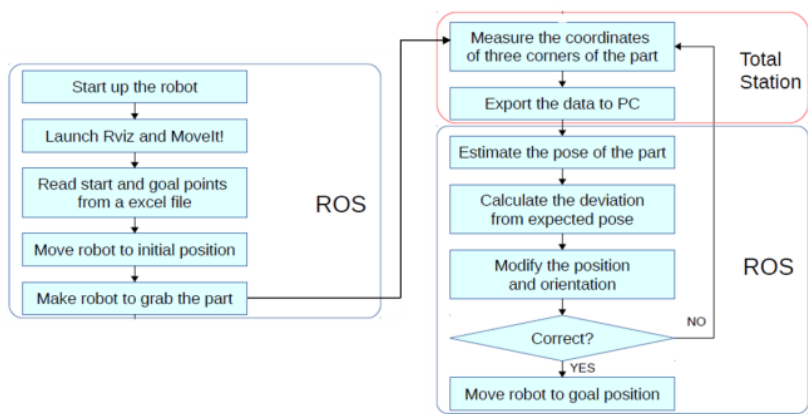

Figure 9. Protocol of the assembly process for the test with the digital theodolite.

For calculating the necessary robotic pose adjustment depending on the element's position, a series of algorithms were used, which is explained as follows.

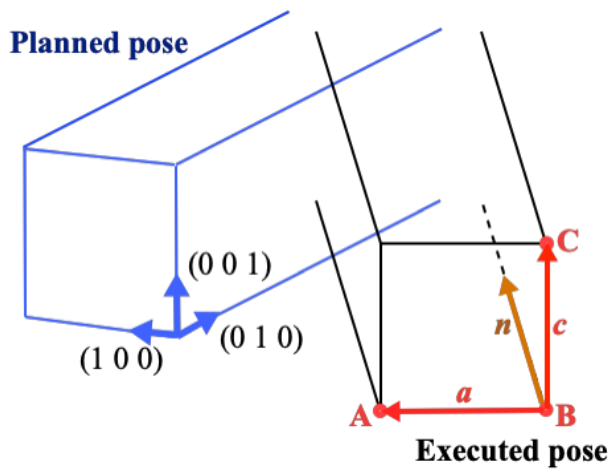

Figure 10. Deviation between the planned pose and the executed pose.

From the three points (in Figure 10, A, B, and C), two vectors of the object were calculated $(\boldsymbol{a}=\mathrm{A}-\mathrm{B}$, $\boldsymbol{c}=\mathrm{C}-\mathrm{B})$. Then, the cross product of the two vectors was the same as the normal vector of them:

$$
\boldsymbol{n}=\boldsymbol{a} \times \boldsymbol{c}
$$

The unit vectors of the three vectors were calculated by dividing by the magnitude of themselves:

$$
\begin{gathered}
\boldsymbol{u}_{a}=\boldsymbol{a} /\|\boldsymbol{a}\| \\
\boldsymbol{u}_{c}=\boldsymbol{c} /\|\boldsymbol{c}\| \\
\boldsymbol{u}_{n}=\boldsymbol{n} /\|\boldsymbol{n}\|
\end{gathered}
$$

Then, the following equation was established:

$$
\begin{aligned}
\left(\begin{array}{lll}
\boldsymbol{u}_{a} & \boldsymbol{u}_{n} & \boldsymbol{u}_{c}
\end{array}\right) & =\boldsymbol{R}_{z} \boldsymbol{R}_{y} \boldsymbol{R}_{x}\left(\begin{array}{ccc}
1 & 0 & 0 \\
0 & 1 & 0 \\
0 & 0 & 1
\end{array}\right) \\
& =\boldsymbol{R}_{z} \boldsymbol{R}_{y} \boldsymbol{R}_{x}
\end{aligned}
$$

where $\boldsymbol{R}_{x}, \boldsymbol{R}_{y}$, and $\boldsymbol{R}_{z}$ are the rotation matrices about x-, $\mathrm{y}$-, and $\mathrm{z}$-axis respectively. When the angles about each axis are $\alpha, \beta$, and $\gamma$;

$$
\begin{aligned}
& \boldsymbol{R}_{z} \boldsymbol{R}_{y} \boldsymbol{R}_{x}= \\
& \left(\begin{array}{ccc}
\mathrm{C} \beta \mathrm{C} \gamma & \mathrm{S} \alpha \mathrm{S} \beta \mathrm{C} \gamma-\mathrm{C} \alpha \mathrm{S} \gamma & \mathrm{C} \alpha \mathrm{S} \beta \mathrm{C} \gamma+\mathrm{S} \alpha \mathrm{S} \gamma \\
\mathrm{C} \beta \mathrm{S} \gamma & \mathrm{S} \alpha \mathrm{S} \beta \mathrm{S} \gamma+\mathrm{C} \alpha \mathrm{C} \gamma & \mathrm{C} \alpha \mathrm{S} \beta \mathrm{S} \gamma-\mathrm{S} \alpha \mathrm{C} \gamma \\
-\mathrm{S} \beta & \mathrm{S} \alpha \mathrm{C} \beta & \mathrm{C} \alpha \mathrm{C} \beta
\end{array}\right)
\end{aligned}
$$

where $C \theta$ means $\cos \theta ; S \theta$ means $\sin \theta$.

Thus, $\boldsymbol{u}_{a}, \boldsymbol{u}_{n}$, and $\boldsymbol{u}_{c}$ were defined as follows:

$$
\left(\begin{array}{lll}
\boldsymbol{u}_{a} & \boldsymbol{u}_{n} & \boldsymbol{u}_{c}
\end{array}\right)=\left(\begin{array}{lll}
u_{a_{x}} & u_{n_{x}} & u_{c_{x}} \\
u_{a_{y}} & u_{n_{y}} & u_{c_{y}} \\
u_{a_{z}} & u_{n_{z}} & u_{c_{z}}
\end{array}\right)
$$

$\alpha, \beta$, and $\gamma$ were calculated by using the following equations:

$$
\begin{gathered}
\alpha=\tan ^{-1}\left(u_{n_{z}} / u_{c_{z}}\right) \\
\beta=\tan ^{-1}\left(-u_{a_{z}} / \sqrt{u_{n_{z}}{ }^{2}+u_{c_{z}}{ }^{2}}\right) \\
\gamma=\tan ^{-1}\left(u_{a_{y}} / u_{a_{x}}\right)
\end{gathered}
$$

On the other hand, the coordinates of the center of the object $\mathrm{P}$ were calculated by the following equation:

$$
\mathrm{P}=\mathrm{B}+(\boldsymbol{a}+\boldsymbol{c}) / 2+l \boldsymbol{u}_{n} / 2
$$

where $l$ is the length of the object.

Then, the deviation of the position and orientation between the planned pose and the executed pose was obtained.

As shown in Figure 11, the robotic system is not complex. However, the interfaces with the digital theodolite were not robust and therefore time consuming.

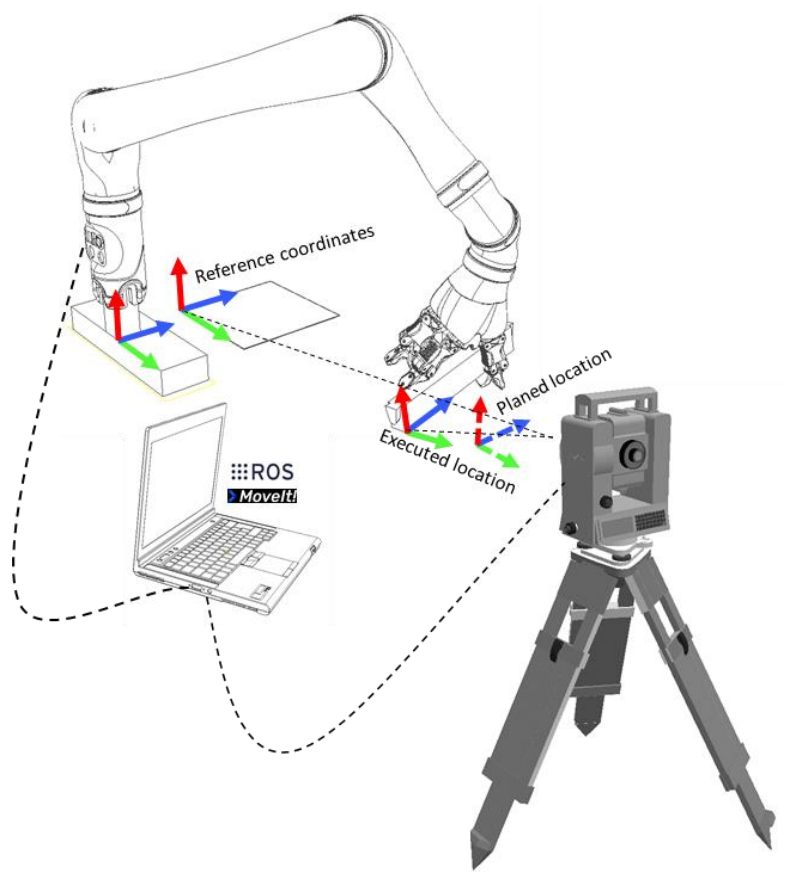

Figure 11. Scheme of the localization of coordinate recognition by using a digital theodolite.

Similar to the test explained in sub-chapter 3.2, there were some issues while estimating the position of the grasped element. The points in this test were selected manually, and therefore the obtained coordinates were subject to errors. 


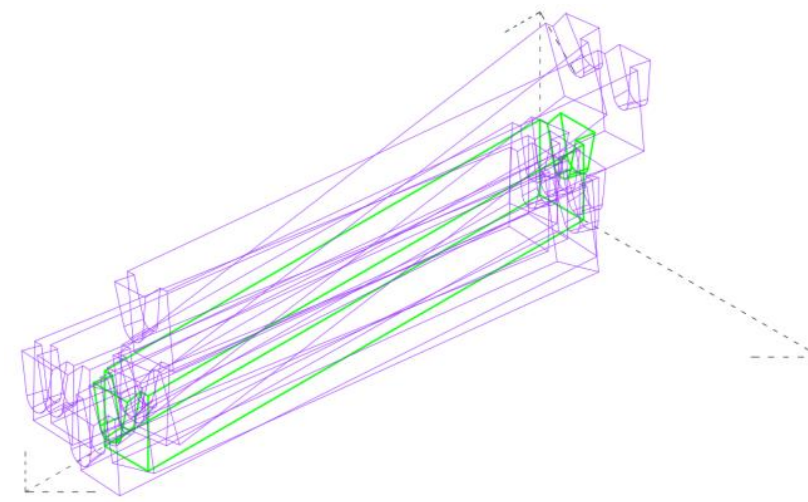

Figure 12. Goal position results by using digital theodolite.

In Figure 12, the five different localizations of the object on the final pose of the robot are shown in purple. In green, the planned location of the object is presented. Table 5 shows the average point coordinates of the five different locations.

Table 5 Absolute location in the test with the digital theodolite $(\mathrm{mm})$ and distance from planned location.

\begin{tabular}{lrrrr}
\hline Name & Position X & Position Y & Position Z & Distance \\
\hline Point 1 & $-6,10$ & 10,60 & 6,90 & 17,09 \\
Point 2 & $-7,90$ & $-18,60$ & 40,70 & 44,43 \\
Point 3 & $-6,40$ & 6,00 & 43,80 & 10,80 \\
Point 4 & $-254,40$ & 26,00 & 5,40 & 7,04 \\
\hline
\end{tabular}

\subsection{Discussion}

The results differ considerably during the three tests. On the first test (without any deviation adjustment), the final pose deviations are too high to accomplish any type of assembly tasks. On the two tests carried out with adjustment operations, the final localization of the object improves considerably, as an average, around $70 \mathrm{~mm}$. The experiments achieved with the guidance of the markers show the best and most accurate results compared to the final desired location of the object. The tests achieved by the coordinate's localization with the digital theodolite present higher deviations than the results gathered with the ArUco markers.

Among the methods presented in this paper, the third test (recognition of coordinates) requires more attention from the human operator. However, it is not necessary to stick a marker on the object to be assembled which, in complex assembly processes where there are many parts, may be advantageous. Human-robot cooperation environments could benefit from this approach as the human worker could mark the necessary points. A specific interface for processing the data gathered with the digital theodolite and linking it with the ROS controller would reduce the time necessary to complete the processing.

Regarding the assembly of large-scale and bended objects such as plaster boards, the markers may be a better solution because the object moves while being handled. This means that the object recognition and its adjustment must be achieved once the object is close to the rest of the assembly.

The results show that, depending on the robotic system's accuracy, there should be consequences in the design of the objects to be assembled. This means that the openness of the joinery system in particular must be dependent on the accuracy of the robotic system. Therefore, in the timber-frame industry, it would be necessary to find the optimal $\mathrm{CNC}$ machining and routing geometries of the objects depending on the robot's assembly accuracy.

\section{Conclusions}

The main problems of assembly processes in fully or partially unstructured environments such as the prefabricated module industry are the inaccuracies associated with the picking and placing of objects. The grasping of objects typically requires structured environments and accurate grasping end-effectors. However, due to the variety of objects, shapes, sizes, and weights in the construction industry, this premise might not always be possible to achieve. In other words, due to the high variety of randomized products and objects in construction and particularly in building renovation, it is difficult to generate a fully structured environment. Therefore, the CNC machined elements of timber-frames need to be recognized before placing them in the module or in the construction site.

In the research outlined in this paper, two techniques have been tested for resolving such inaccuracies. As a conclusion, it can be stated that the objective of the research has been accomplished, which was to adjust the deviations of the grasped objects by recognizing their location. However, future work is necessary to create a more robust solution. Moreover, future experiments will be carried out using 3D laser scanners for localizing the grasped objects' deviations and linking the object with CAD or possibly BIM information.

The procedures executed in this research can be applied to the on-site installation of fully prefabricated modules as well. The pick and place prefabricated modules at the construction site is a task that could benefit from the methods described in this paper.

\section{Acknowledgements}

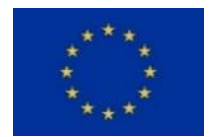

This project has received funding from the European Union's Horizon 2020 research and innovation programme under grant agreement No 636984.

The authors acknowledge the help provided by Mr. Ahmed Agha Osama (from the Chair of Building Realization and Robotics) and by Dr. Peter Wasmeier and the whole Chair of Geodesy of the Technical University of Munich. 


\section{References}

[1] Santamouris, M., Innovating to zero the building sector in Europe: Minimising the energy consumption, eradication of the energy poverty and mitigating the local climate change, Solar Energy, 128 , 61-94, 2016.

https://doi.org/10.1016/j.solener.2016.01.021

[2] Lasarte, N., Chica, J. A., Gomis, I., Benito, J., Iturralde, K., Bock, T., Prefabricated Solutions and Automated and Digital Tools for the optimisation of a Holistic Energy Refurbishment Process. In Proceeding of the 8th EESAP 1st ICAC pages 125140, San Sebastian, Spain, 2017. https://webargitalpena.adm.ehu.es/pdf/UCPDF176683.pdf

[3] Iturralde, K., Linner, T., Bock, T. First Monitoring and Analysis of the Manufacturing and Installation Process of Timber Based 2D Modules for Accomplishing a Future Robotic Building Envelope Upgrading. In Proceedings of ISARC 2017, pages 65-73, Taipei, 2017. https://doi.org/10.22260/ISARC2017/0009

[4] Robeller, C., Konakovic, M., Dedijer, M., Pauly, M. and Weinand, Y., A Double-Layered Timber Plate Shell-Computational Methods for Assembly, Prefabrication, and Structural Design. In Proceedings of Advances in Architectural Geometry, pages 104-122. Zürich, Switzerland 2016. https://vdf.ch/advances-in-architecturalgeometry-2016-e-book.html

[5] Braumann, J., Brell-Çokcan, S., Parametric Robot Control: Integrated CAD/CAM for Architectural Design. In Proceedings of the 31st annual conference of the Association for Computer Aided Design in Architecture, pages 242-251, Calgary, Canada, 2011. https://publications.rwthaachen.de/record/658507

[6] Willmann, J., Knauss, M., Bonwetsch, T., Apolinarska, A.A., Gramazio, F. and Kohler, M., Robotic timber construction-Expanding additive fabrication to new dimensions, Automation in construction,61:16-23,2016.

https://doi.org/10.1016/j.autcon.2015.09.011

[7] Kodama, Y., Yamazaki, Y., Kato, H., Iguchi, Y., Naoi. H., A robotized wall erection system with solid components, In Proceedings of the 5th ISARC, Pages 441-448. Tokyo, Japan 1988. https://doi.org/10.22260/ISARC1988/0052

[8] Bock, T., A Study on Robot-Oriented Construction and Building System. Ed: University of Tokyo, In: National Institute of Informatics, Tokyo, 1989.

[9] Gambao, E., Balaguer, C., Barrientos, A., Saltaren, R, Puente, E.A., Robot assembly system for the construction process automation, In Proceedings of International Conference on Robotics and Automation, pages 46-51, Albuquerque, USA, USA. http://ieeexplore.ieee.org/stamp/stamp.jsp?tp=\&ar number $=620014$ \&isnumber $=13464$

[10] Bonwetsch, T., Robotically assembled brickwork: manipulating assembly processes of discrete objects, PhD diss., ETH Zurich, 2015.

[11] Randek Zero labor. http://www.randek.com/en/wall-floor-and-roofproduction-lines/zerolabor, On-line: 28/01/2019

[12] Iturralde, K. Bock, T., Integrated, Automated and Robotic Process for Building Upgrading with Prefabricated Modules, In Proceedings of the 35th ISARC, pages 340-347 , Berlin, Germany, 2018. https://doi.org/10.22260/ISARC2018/0048

[13] Maropoulos, P. G., Muelaner, J. E., Summers, M. D., Martin, O. C., A new paradigm in large-scale assembly-research priorities in measurement assisted assembly, The International Journal of Advanced Manufacturing Technology, 70(1-4), 621-633, 2014 https://doi.org/10.1007/s00170-013$\underline{5283-4}$

[14] Drouot, A., Zhao, R., Irving, L., Sanderson, D. and Ratchev, S., Measurement assisted assembly for high accuracy aerospace manufacturing, IFACPapersOnLine, 51(11), pp.393-398, 2018 https://doi.org/10.1016/j.ifacol.2018.08.326

[15] Nottensteiner, K., Bodenmueller, T., Kassecker, M. Roa, M.A., Stemmer, A., Stouraitis, T., Seidel, D. and Thomas, U., A complete automated chain for flexible assembly using recognition, planning and sensor-based execution. In Proceedings of $47 \mathrm{st}$ International Symposium on Robotics; pages 1-8, Munich,Germany 2016 https://ieeexplore.ieee.org/document/7559140

[16] Feng, C., Xiao, Y., Willette, A., McGee, W. and Kamat, V.R., Vision guided autonomous robotic assembly and as-built scanning on unstructured construction sites, Automation in Construction, 59, 128-138,2015. https://doi.org/10.1016/j.autcon.2015.06.002

[17] Lundeen, K.M., Dong, S., Fredricks, N., Akula, M., Seo, J. and Kamat, V.R., Optical marker - based end effector pose estimation for articulated excavators, Automation in Construction, 65, 51-64, 2016. https://doi.org/10.1016/j.autcon.2016.02.003

[18] https://dynamobim.org/, Accessed: 28/01/2019

[19] Open Source Computer Vision Library, https://opencv.org/, On-line: 28/01/2019

[20] Oberkampf, D., DeMenthon, D.F. and Davis, L.S., Iterative pose estimation using coplanar feature points, Computer Vision and Image Understanding, 63(3), 495-511, 1996. https://doi.org/10.1006/cviu.1996.0037 